treatment plan included neoadjuvant CRT (cisplatin + 5-Fluorouracil/45 Gy) followed 6-8 weeks later by a transthoracic en bloc oesophagectomy. Morphological evaluations combined with FDGPET results were performed 2 weeks before and $4-6$ weeks after the completion of CRT. Intratumoural pre- and post-treatment FDGstandardised uptake values were assessed (SUV1, SUV2, percentage change). These variables were correlated with pathologic and morphologic responses and survival. Investigators were blinded to the FDG-PET results unless metastatic disease was suspected.

Results Out of 60 total patients, 46 underwent the complete treatment plan (median age: 60.1 years; adenocarcinoma: 25 patients; squamous cell cancer: 21 patients). A major pathological response occurred in $19.6 \%$ of patients and was associated with a favourable outcome $(p=0.057)$. Neoadjuvant CRT led to a significant reduction in intratumoral FDG-uptake $(p<0.001)$. No significant association was seen between a pathologic response (either complete or major) and the FDG-PET results ( $p>0.280)$. The SUV2 value was correlated with a morphological response and the possibility to perform an R0 resection $(p<0.018$; ROC analysis: SUV2 threshold $=5.5$ ). No significant association was found between metabolic imaging and recurrence or survival.

Conclusion FDG-PET does not effectively correlate with pathologic response and long-term survival in patients with locally advanced oesophageal cancer undergoing neoadjuvant CRT followed by surgery (registered on http://www.e-cancer.fr website, RECF0350, 2002-1936R).

Funding Funded by the French ministry of Health - Programme Hospitalier de Recherche Clinique 2002.

Competing interests None declared.

\section{PTU-184 DEFINING AND TREATING A POSITIVE CIRCUMFERENTIAL RESECTION MARGIN IN OESOPHAGEAL AND GASTRO-OESOPHAGEAL JUNCTIONAL CANCER}

doi:10.1136/gutjnl-2012-302514c.184

1J R O'Neill, ${ }^{1} \mathrm{~N}$ Stephens, ${ }^{2} \mathrm{~V}$ Save, ${ }^{3} \mathrm{H}$ Phillips, ${ }^{2} \mathrm{H} \mathrm{M}$ Kamel, ${ }^{1} \mathrm{~A}$ G Robertson, ${ }^{4} \mathrm{P}$ J Driscoll, 'S Paterson-Brown. 'General Surgery, Royal Infirmary of Edinburgh, Edinburgh, UK; ${ }^{2}$ Pathology, Royal Infirmary of Edinburgh, Edinburgh, UK; ${ }^{3}$ Oncology, Western General Hospital, Edinburgh, UK; ${ }^{4}$ General Surgery, Queen Margaret Hospital, Dunfermline, UK

Introduction A positive circumferential resection margin (CRM) has been implicated with poorer prognosis in oesophageal and gastrooesophageal junctional (OGJ) cancer. The Royal College of Pathologists (RCP) defines a margin as positive if tumour cells are present within $1 \mathrm{~mm}$. In contrast, the College of American Pathologists (CAP) only defines a margin as positive if tumour cells are observed at the margin. The equivalence of the systems is not clear and the impact of adjuvant treatment has not been assessed.

Aims To compare the prognostic ability of the RCP and CAP systems in a cohort from a single UK centre and to determine if adjuvant radiotherapy offers a survival benefit for CRM positive patients.

Methods Patients with a "T3" adenocarcinoma or squamous cell carcinoma of the oesophagus or OGJ undergoing potentially curative resection between 1994 and 2010 were identified from a prospective database. Resection specimens were reviewed and the CRM was measured to $\pm 0.1 \mathrm{~mm}$ by a consultant pathologist. Univariate, multivariate and propensity score matching analyses (PSMA) were performed.

Results A total of 226 patients were included. Cox regression demonstrated patient sex $(p=0.009)$, tumour differentiation $(p=0.015)$, nodal $(N)$ stage $(p<0.001)$ and CRM group $(p=0.045)$ were independently predictive of prognosis. Patients were grouped into CRM of $0 \mathrm{~mm}$ (CAP+ve, $\mathrm{n}=47)$, CRM $>0 \mathrm{~mm}$ but $<1 \mathrm{~mm}$
(RCPCRM, $\mathrm{n}=83$ ) and CRM $\geq 1 \mathrm{~mm}$ (CRM-ve, $\mathrm{n}=96$ ). Median survivals ( $95 \% \mathrm{CIs})$ were significantly different across groups $(p=0.019)$ with CAP+ve $=18$ months (13.0 to 23.0), RCPCRM $=$ 28 months (18.6 to 37.3) and CRM-ve $=33$ months (25.8 to 40.2). A trend for poorer survival was noted for the CAP+ve vs the RCPCRM group $(p=0.073)$ although there was heterogeneity in $\mathrm{N}$ stage across groups. PSMA demonstrated no residual survival difference between CAP+ve and RCPCRM groups when other prognostic variables were controlled. Significant selection bias was observed for patients undergoing adjuvant radiotherapy. PSMA was applied to assess the treatment effect. Patients undergoing adjuvant radiotherapy $(n=23)$ showed significantly improved survival when compared to controls $(n=23)$ matched for sex, pre-operative treatment, $\mathrm{N}$ stage, histology and differentiation $(\mathrm{p}=0.04)$.

Conclusion The survival difference between CAP+ve and RCPCRM groups could be explained by existing prognostic variables. The CAP and RCP systems therefore appear equivalent in our cohort. In selected patients with a CRM $<1 \mathrm{~mm}$, adjuvant radiotherapy may be of benefit and a prospective randomised trial is indicated.

Competing interests None declared.

\section{PTU-185 NOVEL TECHNIQUES FOR ASSESSING OESOPHAGO- PHARYNGEAL REFLUX IN PATIENTS WITH HOARSENESS AND SUSPECTED LARYNGOPHARYNGEAL REFLUX}

doi:10.1136/gutjnl-2012-302514c.185

${ }^{1,2} \mathrm{~J} O$ Hayat, ${ }^{*}{ }^{2} \mathrm{E}$ Yazaki, ${ }^{3} \mathrm{~A}$ T Moore, ${ }^{3} \mathrm{~L}$ A C Hicklin, ${ }^{4} \mathrm{P}$ W Dettmar, ${ }^{1} \mathrm{~J}-Y$ Kang, ${ }^{2} \mathrm{D}$ Sifrim. ' Gastroenterology, St. George's Hospital, London, UK; ${ }^{2}$ GI Physiology, Barts and the London School of Medicine and Dentistry, London, UK; ${ }^{3}$ ENT Surgery, St. George's Hospital, London, UK; ${ }^{4}$ Technostics, Kingston-upon-Hull, UK

Introduction It is suggested that hoarseness along with typical signs on laryngoscopy can be caused by oesophago-pharyngeal reflux, often referred to as LPR. New methods are proposed to assess pharyngeal exposure to gastric contents. They are suggested to measure (1) liquid or mixed gas-liquid acid and non-acid reflux (HMII-pH), (2) aerosolized acid reflux (Dx-pH measuring system, Restech), and (3) presence of pepsin in saliva. We aimed to quantify pharyngeal exposure to gastric contents in patients with hoarseness and healthy controls using the above techniques.

Methods 21 patients with hoarseness and a positive laryngoscopy (mean age: 51 range: $23-75$ ) and 10 asymptomatic controls (mean age: 26, range: 21-34) underwent simultaneous HMII-pH monitoring, oropharyngeal $\mathrm{pH}$ monitoring and saliva pepsin sampling. The HMII-pH catheter was located with impedance sensors in the oesophageal body, $3-5 \mathrm{~cm}$ distal and $0-2 \mathrm{~cm}$ proximal to the UOS. The Dx-pH catheter was located posterior to the uvula and pepsin in saliva was measured using an in vitro device utilising two pepsin monoclonal antibodies (PepTest) at five different times during the 24-h period. Patients were studied "off" PPI.

Results Healthy controls had (1) no liquid or mixed gas/liquid reflux in the pharynx, (2) two controls had +ve Dx-pH and (3) two controls had more than one saliva sample +ve for pepsin with the other tests negative. Patients were classified into four groups: (a) all tests +ve $(n=2)$; (b) two tests +ve (MII-pH + pepsin $(n=5)$ or MII$\mathrm{pH}+\mathrm{Dx}-\mathrm{pH}(\mathrm{n}=3) ;(\mathrm{c})$ all tests negative $(\mathrm{n}=5)$ and $(\mathrm{d})$ patients with +ve Dx-pH or pepsin without evidence of HMII detected reflux. These patients were considered negative $(n=6)$. Dx-pH drops were poorly associated with HMII-pH reflux. $11 \%$ of $\mathrm{Dx}-\mathrm{pH}$ drops to $\mathrm{pH}<4,15 \%$ of $\mathrm{pH}$ drops to $\mathrm{pH}<5$ and $10 \%$ of $\mathrm{pH}$ drops to $\mathrm{pH}<5.5$ coincided with HMII detected liquid or gas reflux in the oesophageal body. The detection of pepsin in saliva occurred in $7 / 10$ patients with acid or non-acid HMII detected reflux. Positive pepsin saliva samples were preceded by more reflux events in the previous 60 min $3(1-4)$ than negative samples $0(0-2) \mathrm{p}<0.0001$ 